\title{
Postural phenotype assessment in pupils: a new clinic approach
}

\author{
Luana Karina de Almeida Nascimento', Cristiano Barreto de Miranda', Flávio Martins do Nascimento Filho', \\ Felipe Lima de Cerqueira², Maria das Graças Rodrigues de Araújo³ ${ }^{3}$ Miburge Bolívar Gois Júnior
}

\begin{abstract}
Introduction: Most of the postural problems originate during puberty, when the body structure is in the process of development and the hormonal release predisposes the differentiated growth between the gender, which can interfere in the posture and balance of the individual. Objective: To analyze the postural phenotypic parameters in school adolescents. Method: A cross-sectional study was carried out in 72 adolescents with age between 10 and 15 years from a public school in the city of Lagarto, Sergipe. The subjects were divided into two groups according to gender (G1: female group; G2: male group) and age subgroups (SB1: adolescents with 10-12 years; SB2: adolescents with 13-15 years), homogeneous in relation to BMI. The research consisted of the collection of anthropometric and clinical data, backpack weighing, static postural evaluation through biophotogrammetry and measurement of stabilometric parameters through a force platform. Results: The individuals presented reduced mobility of the spine and did not overload the school backpack. The prevalence of scoliosis and scoliotic attitudes was 33.32\% and more frequentily in the G2. As for the postural angles, the G1 presented knee valgus, hyperextension of the knee and lumbar hyperlordosis, differently, the G2 was characterized with forward inclination of the body, knee flexion, cervical hyperlordosis and thoracic hyperkyphosis. There was no difference in body balance variables between gender, besides the correlation between postural alignment and balance. Conclusion: The study suggests that the phase of corporal development in the school period favors postural phenotypic differentiations.
\end{abstract}

Keywords: Posture; Body Balance; Adolescent; Gender

\section{INTRODUCTION}

Puberty is considered a phase of marked bodily transformations, due to the accelerated musculoskeletal development, axial growth and hormonal determinants that allow the maturation and differentiation between girls and boys. ${ }^{(1)}$ It is configured in parallel, a period of intense technological experience due to the abusive use of electronic media ${ }^{(2-4)}$ and formation of the corporal scheme through the influences of the school environment. ${ }^{(5)}$ Together, these factors are reported as predictive of the onset and progression of postural disorders, especially affecting the spine. ${ }^{(6-8)}$

Studies strive to compare postural alignment between the gender. ${ }^{(9-12)}$ However, human posture represents a complex mechanism approaching the individual as a whole, thus a deep understanding of the postural system in adolescents is still needed. Exposure to overloads through the use of school backpacks ${ }^{(13,14)}$ and remain in a sitting position for longer periods of time ${ }^{(15)}$ is an important aggravating factor with adverse effects on health, affecting the joint mobility of the spine, the appearance of scoliotic curves, two-dimensional postural deviations and changes in body balance.
We present, in an original way, the term postural phenotype, formed by the interaction between posture, adaptive strategies of balance, environmental influences and body changing conditions, using a clinical screening protocol and approaching the postural balance assessment, its modifiers and relationships. The objective of this study was to analyze postural phenotypic parameters in school adolescents, measuring the differences between the genders. It is assumed that these results may be useful for a better understanding of sexual specificities during the growth period implied globally in postural health.

\section{METHOD}

\section{Participants}

An analytical cross-sectional study was carried out with 72 healthy individuals, 37 girls and 35 boys from a public school in the city of Lagarto, Sergipe, Brazil.

Subjects, with age between 10 and 15 years, were voluntarily recruited, since they were not using anti-inflammatory, antibiotics, anticonvulsants, oral contraceptives and

\footnotetext{
Corresponding Author: Luana Karina de Almeida Nascimento. Endereço: LCMEP- (Laboratório de Controle Motor e Equilíbrio Postural), Post-Graduate Program in Health Science - Federal University of Sergipe- UFS/Lagarto. Antônio Garcia Filho, Av. Governador Marcelo Déda S/N, Centro, Lagarto (SE), Brazil. Zip Code: 49400-000, Phone: +55 (79) 88675490. E-mail: ft.luanakarina@gmail.com

1,2 Member of Motor Control and Postural Balance Laboratory, Universidade Federal de Sergipe (UFS), Lagarto (SE), Brazil.
} 
adjuvants medication, did not present any pathologies, deformities, orthopedic and/or neurological post-surgical procedures, visual, auditory and/or somatosensory deficits or using orthoses and/or prostheses. Free and informed consent ratified by the Ethics Committee of the Universidade Federal de Sergipe ( $n$ o 903.958) was obtained from all those responsible.

For comparison purposes, the groups were divided according to gender (G1=Female group, G2=Male group) and in case of statistically significant difference they were stratified by age group through a categorization according to the stages of growth of each gender, considering the SB1 individuals between $10-12$ years and the SB2 to those between 13-15 years.

\section{Screening Procedures}

A screening was carried out in order to investigate only eutrophic individuals. The measurement of the Body Mass Index (BMI) was then determined, and the weight was measured in $\mathrm{kg}$ by the Serene ${ }^{\circledR}$ digital scale and the height was measured in $\mathrm{cm}$ by the Welmy ${ }^{\circledR}$ stadiometer. For the measurements, the subjects positioned themselves with parallel and bare feet, arms relaxed next to the body and wearing light clothes. The BMI was calculated using as reference the percentile curves for gender and age, considering the rankings equivalent to the normal BMI ( $\geq$ percentile 3 and $<$ percentile 85) according to the World Health Organization (WHO). ${ }^{(16)}$

\section{Measurement of clinical modifiers}

The mobility of the lumbar spine was evaluated through the Modified Schober's test, with the individual in standing position and with the trunk region free of clothing for the location of the 5 th lumbar vertebra (L5), where they were marked $5 \mathrm{~cm}$ below and $10 \mathrm{~cm}$ above this reference. Then, an anterior trunk flexion was performed, keeping knees extended. The evaluator determined the distance between the two points marked along the column with a tape measure, and were considered normal the one that exceeded at least $5 \mathrm{~cm}$ from the initial measurement.

Then, the examination was performed to investigate the axial thoracic and lumbar rotation by the Ortophaedics Systems Incorporation ${ }^{\circledR}$ escoiometer. The spinous processes of T6 and $\mathrm{L} 3$ were demarcated and the individual reproducing the Adams forward bend test had the scoliometer positioned over the respective spinous processes, perpendicular to the axial plane, in which the complete stop of the mercury ball signaled the precise angulation in Cobb degrees. For interpretation of the test, two-dimensional deviations below 10 Cobb were classified as scoliotic attitudes and above $10 \circ$ Cobb as scoliosis.

Information on the transportation of school supplies was also collected, specifically investigating the weight of the backpack, adopting WHO regulations in which the load of backpacks, briefcases and similar should not exceed $10 \%$ of the body weight of the elementary school student.

\section{Posture Evaluation}

With the individual in intimate clothing and without accessories in the body, anatomical points were identified for postural evaluation by the PAS ${ }^{\circledR}$ (Postural Evaluation Software). To do this, were used Styrofoam balls sized $15 \mathrm{~mm}$, and fixed by adhesive tape to correctly mark the anatomical points, which followed the PAS protocol of measurements ${ }^{17}$ to analyze anterior (Figure 1), posterior (Figure 2) and lateral (Figure 3) view and for the evaluation of the vertebral curvatures were marked the spinous processes of C7, T1, T12, L1 and L5.

The individual was then positioned on a rubber mat, where the contour of the feet was performed, which ensured a single standing position for photography in all views. In order to record the images, was used a Canon PowerShot G11 ${ }^{\circledR}(10 \mathrm{MP})$ photographic camera, positioned 3 meters away from the subject, fixed on a professional tripod and a plumb line for calibration of the photo in the biophotogrammetric program.

The analysis of postural alterations was performed using the PAS $^{\circledR}$ biophotogrammetric method by which were obtain angles of the anatomical markings. For the interpretation of the angles formed in the frontal plane we considered the negative value as left slope, positive value as right slope and the alignment

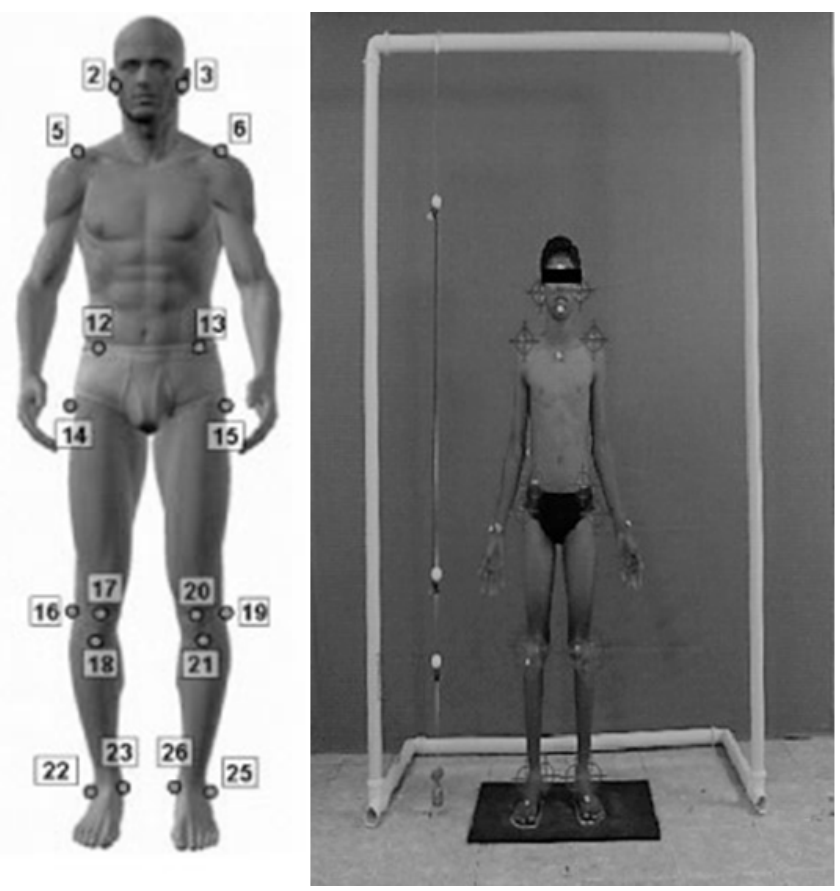

Figure 1: Anatomic points of the anterior view of PAS protocol ${ }^{17}$. Legend: 2 right ear wolf; 3 left ear wolf; 5 right acromion; 6 left acromion; 12 right anterior-superior iliac spine; 13 left anterior-superior iliac spine; 14 right femur greater trochanter; 15 left femur greater trochanter; 16 right knee articular line; 17 right patella medium point; 18 right tibia tuberosity; 19 left knee articular line; 20 left patella medium point; 21 left tibia tuberosity; 22 right lateral malleolus; 23 right medial malleolus; 25 left lateral malleolus; 26 left medial malleolus. 


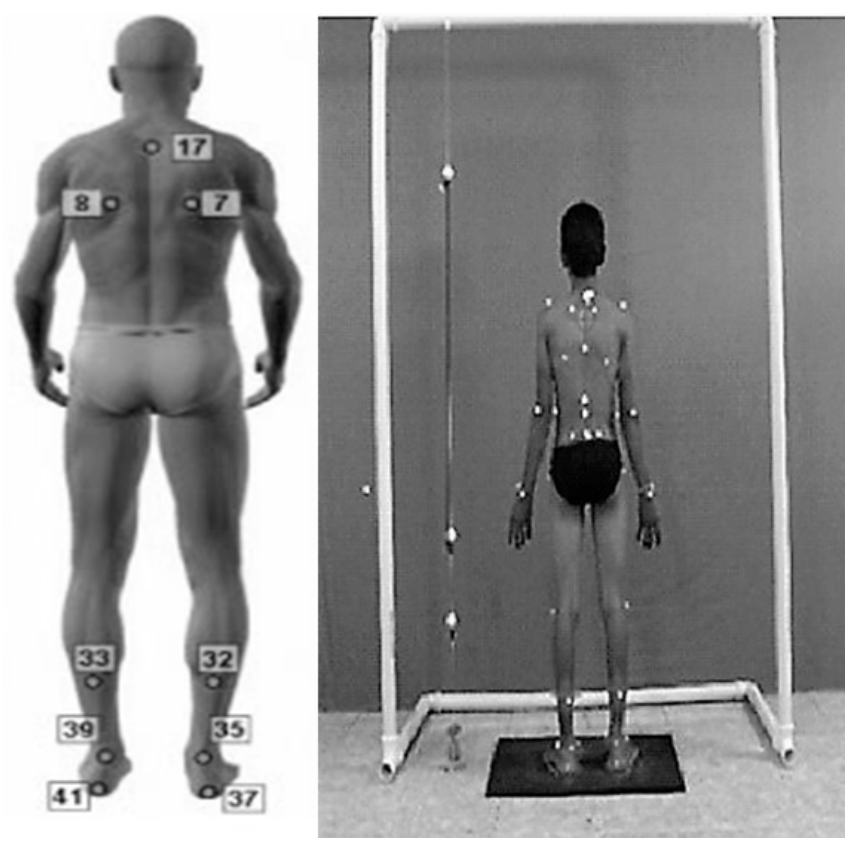

Figure 2: Anatomic points of the posterior view of PAS protocol ${ }^{17}$. Legend: 17 spinal process of T3; 7 right scapula inferior angle; 8 left scapula inferior angle; 32 right leg point medial line; 33 left leg poit medial line; 35 right calcaneum tendon between malleolus; 39 left calcaneum tendon between malleolus; 37 right calcaneum; 41 left calcaneum.

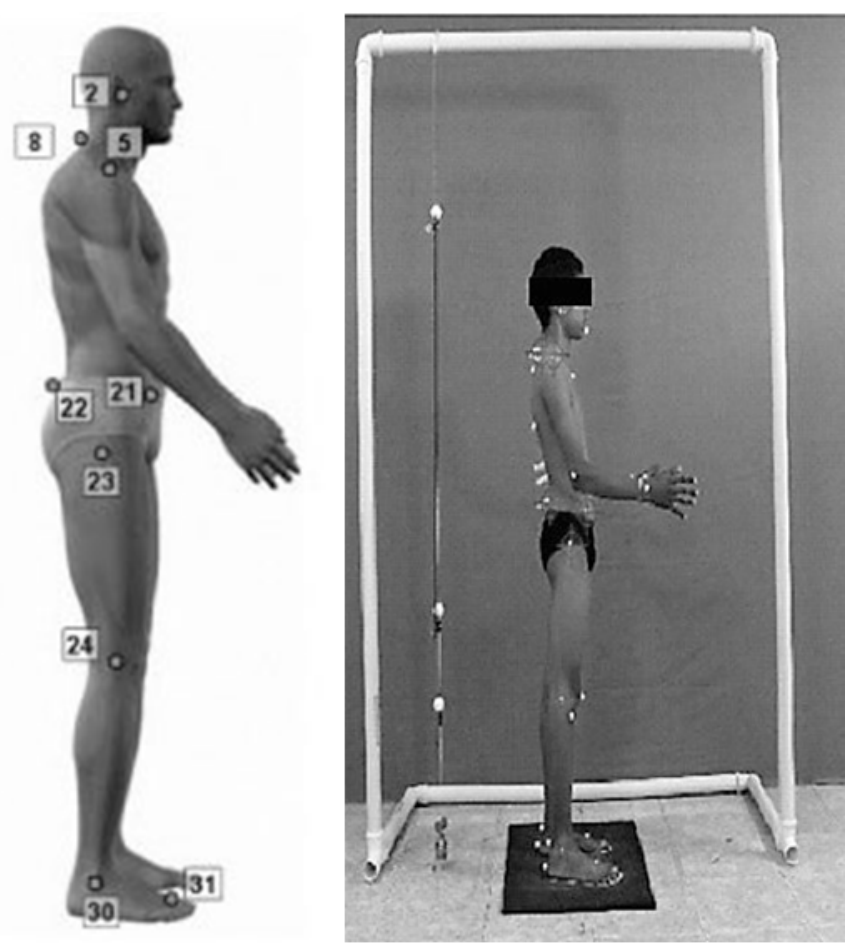

Figure 3: Anatomic points of the lateral view of PAS protocol ${ }^{17}$. Legend: 2 right ear wolf; 5 right acromion; 8 spinal process of C7; 21 right anterior-superior iliac spine; 22 right posterior-superior iliac spine; 23 right femur greater trochanter; 24 right knee articular line; 30 right lateral malleolus; 31 medium point between second and third right metatarsus. represented by the zero value. For the $Q$ and ankle angles, the normative was established in 15 and 5 degrees respectively.

With regard to the angles in the lateral views, were adopted classifications: head alignment in extension and flexion; anterior and posterior position for pelvic tilt and hip angle; anterior and posterior inclination for the body alignment; flexion and hyperextension in the knee alignment; and values of the ankle positioning represented plantar flexion and dorsiflexion. Regarding the angles of the vertebral curvatures, it was considered that the greater the angle the greater the rectification and the smaller the angle the greater the curvature.

\section{Balance Evaluation}

For the measurement of the static body balance through stabilometric parameters was used a Footwork ${ }^{\circledR}$ force platform (version 3.2.2.0). The analysis was performed with the individual in bipodal support, bare feet, assuming a comfortable and usual posture, oriented to remain on the platform without moving or talking during the evaluation period, which comprised 20 seconds of preparation for recognition of the plantar impressions and approach of the points of pressure center and barycentre and in 10 seconds stabilometry is measured, allowing information on the center of pressure (CoP), oscillation in the anterior-posterior (OAP) and medial-lateral (OML) directions.

\section{Statistical analysis}

The Shapiro-Wilk test was used to study the variables normality. The analysis of the comparison between the clinical variables weight, BMI, Schober, axial thoracic and lumbar rotation was obtained using the Mann-Whitney test and to compare the height and weight of the backpack was applied independent Student's T test. For comparisons of posture and balance variables, independent Student t and Mann-Whitney tests were considered. Regarding the correlation analysis between postural angles and the variables of COP, OAP and OML were applied the Pearson and Spearman Correlation tests. For all analyzes, were used the Statistical Package for the Social Sciences (Inc., Chicago, USA; SPSS 15.0) and the statistical significance stipulated in $5 \%(p<0.05)$ and $\mathrm{Cl}$ of $95 \%$.

\section{RESULTS}

The age presented median of 12 (11-12) years in the G1 and $12(11-13)$ years in the G2. There was no statistically significant difference between age and gender $(p=0.911)$ and the same for the BMI $(p=0.844)$. Sampling homogeneity was also present in the results of clinical modifiers, with decreased joint mobility in both groups $(p=0.887)$ and no overload in the backpack $(p=0.518)$. The prevalence of scoliosis and scoliotic attitudes was 33.32\% (G1=15.32\%; G2=18.00) and high rates of scoliotic attitudes were observed in the thoracic and lumbar level (Table 1). 
Postural analysis in the frontal plane allowed the visualization of a statistically significant difference between groups for ÂFMId ( $p=0.042)$, ÂFMle ( $p=0.006)$, ÂQd $(p=0.001)$ and $\hat{A} Q$ e $(p=0.001)$ angle in the anterior view, However, no significant differences were found for angles in the posterior view (Table 2). In sagittal plane, were found statistical differences in the ALICORP $(p=0.015)$ and ÂJOEL $(p=0.017)$ angle in the left lateral view (Table 3), as well as for all angles of vertebral curvature (Table 4). Clarifying the differences found, the analysis of the age subgroups found a statistical difference in the Âqd, Âqe, ÂC and ÂT angles (Table 5), demonstrating that SB1 develops pronounced knee angulations in comparison to SB2, whereas the opposite occurs in cervico-thoracic curvatures. Despite this, no statistical difference was found in the comparison of the balance variables (Table 6). There was no correlation between posture and balance.

\section{DISCUSSION}

The term postural phenotype integrates the actual perception of postural variants and balance, considering the clinical modifiers that act in this mechanism of body readjustment in face of the new corporal proportions of the adolescent. Dolphens et al. ${ }^{(12)}$, through a study that proposed to measure the differences between the sexes during the pubertal period considering different types of posture, verified anterior inclination of the body, thoracic kyphosis and more pronounced pelvic anteversion in boys when compared to the girls, coinciding with our findings. They also speculated that this characteristic may be a consequence of increased muscle recruitment and hypertrophy of the trunk extensors, resulting in decreased levels of flexibility parallel to the force gain, allowing the development of ankylosing spondylitis (AS),

Table 1 - Description of anthropometric and clinical variables.

\begin{tabular}{|c|c|c|c|}
\hline Variable & G1 & G2 & $P$ value \\
\hline Weight & $41.10(35.40-44.62)$ & $37.20(33.10-47.80)$ & 0.330 \\
\hline Height & $1.47 \pm 0.09$ & $1.45 \pm 0.11$ & 0.464 \\
\hline BMI & $18.31(16.61-20.23)$ & $18.03(16.66-20.69)$ & 0.844 \\
\hline Joint Mobility & $4.30(3.50-4.50)$ & $4.00(3.50-4.70)$ & 0.887 \\
\hline Thoracic axial rotation & $4.00(2.00-10.00)$ & $8.00(4.00-10.00)$ & 0.235 \\
\hline Lumbar axial rotation & $8.00(4.00-10.00)$ & $10.00(4.00-12.00)$ & 0.152 \\
\hline Backpack weight & $3.00 \pm 0.89$ & $2.84 \pm 1.21$ & 0.518 \\
\hline
\end{tabular}

BMI (Body Mass Index), G1 (Female Group), G2 (Male Group)

Table 2 - Description of posture variables in anterior and posterior views.

\begin{tabular}{|c|c|c|c|}
\hline Variable & G1 & G2 & $p$ value \\
\hline \multicolumn{4}{|l|}{ Anterior view } \\
\hline ALICAB & $0.00(-2.10-3.00)$ & $-0.60(-2.50-2.90)$ & 0.897 \\
\hline ALIACRO & $-0.61 \pm 1.89$ & $-0.17 \pm 1.67$ & 0.298 \\
\hline ALIEIAS & $-0.44 \pm 2.75$ & $-0.25 \pm 3.19$ & 0.789 \\
\hline ÂACREIAS & $0.17 \pm 2.87$ & $-0.10 \pm 3.51$ & 0.713 \\
\hline ÂFMId & $-2.27 \pm 2.87$ & $-0.98 \pm 2.36$ & $0.042^{*}$ \\
\hline ÂFMle & $-2.87 \pm 2.88$ & $-0.97 \pm 2.75$ & $0.006^{*}$ \\
\hline ALIHTT & $0.24 \pm 2.74$ & $0.91 \pm 2.53$ & 0.290 \\
\hline$\hat{A} Q d$ & $29.41 \pm 11.50$ & $18.38 \pm 6.31$ & $0.001^{*}$ \\
\hline ÂQe & $28.30(21.75-41.55)$ & $18.90(14.70-22.60)$ & $0.001 *$ \\
\hline \multicolumn{4}{|l|}{ Posterior view } \\
\hline ASSESC & $2.59 \pm 22.20$ & $4.92 \pm 23.73$ & 0.669 \\
\hline ÂRETd & $6.70(3.10-13.50)$ & $8.50(1.40-12.20)$ & 0.960 \\
\hline ÂRETe & $10.80(2.90-14.50)$ & $9.10(1.80-13.80)$ & 0.804 \\
\hline
\end{tabular}


Table 3 - Description of posture variables in right and left lateral views.

\begin{tabular}{|c|c|c|c|c|}
\hline & & G1 & G2 & $P$ value \\
\hline \multirow{2}{*}{ ALIC7 } & $\mathrm{R}$ & $50.50(46.55-52.70)$ & $52.60(46.40-56.70)$ & 0.161 \\
\hline & $\mathrm{L}$ & $50.12 \pm 5.51$ & $51.40 \pm 6.19$ & 0.357 \\
\hline \multirow{2}{*}{ ALICABE } & $\mathrm{R}$ & $10.12 \pm 8.02$ & $8.82 \pm 8.82$ & 0.515 \\
\hline & $\mathrm{L}$ & $9.63 \pm 6.82$ & $8.90 \pm 8.70$ & 0.689 \\
\hline \multirow{2}{*}{ ÂQUADIL } & $\mathrm{R}$ & $-12.00(-15.00--8.15)$ & $-10.20(-14.20--5.60)$ & 0.226 \\
\hline & $\mathrm{L}$ & $-11.90(-16.95-6.15)$ & $-8.90(-12.10-5.10)$ & 0.119 \\
\hline \multirow{2}{*}{ ALICORP } & $\mathrm{R}$ & $1.28 \pm 1.44$ & $1.84 \pm 1.70$ & 0.141 \\
\hline & $\mathrm{L}$ & $0.97 \pm 1.18$ & $1.87 \pm 1.81$ & $0.015 *$ \\
\hline \multirow{2}{*}{ ALIPELV } & $\mathrm{R}$ & $-8.69 \pm 5.77$ & $-9.37 \pm 7.70$ & 0.672 \\
\hline & $\mathrm{L}$ & $-9.20 \pm 6.45$ & $-10.12 \pm 7.14$ & 0.567 \\
\hline \multirow{2}{*}{ ÂJOEL } & $\mathrm{R}$ & $-1.45 \pm 4.94$ & $0.91 \pm 5.60$ & 0.050 \\
\hline & $\mathrm{L}$ & $-2.09 \pm 4.98$ & $0.87 \pm 5.35$ & $0.017^{*}$ \\
\hline \multirow{2}{*}{ ÂTORN } & $\mathrm{R}$ & $85.94 \pm 3.96$ & $84.86 \pm 4.01$ & 0.251 \\
\hline & $\mathrm{L}$ & $85.85 \pm 4.10$ & $85.03 \pm 5.05$ & 0.447 \\
\hline
\end{tabular}

G1 (Female Group), G2 (Male Group), R (Right), L (Left), ALIC7 (Horizontal alignment of the head -C7), ALICABE (Vertical alignment of the head - acromion), ÂQUADIL (Hip angle - trunk and thigh), ALICORP (Vertical alignment of the body), ALIPELV (Horizontal alignment of the pelvis), ÂJOEL (Knee angle), ÂTORN (Ankle angle).

Table 4 - Description of the posture variables related to the angles of the vertebral curvatures.

\begin{tabular}{cccc}
\hline Variable & G1 & G2 & P value \\
\hline ÂC & $40.12 \pm 6.18$ & $34.41 \pm 6.29$ & $\mathbf{0 . 0 0 1 ^ { * }}$ \\
$\hat{A} T$ & $42.60(38.75-51.30)$ & $38.40(33.90-40.90)$ & $\mathbf{0 . 0 0 3 *}^{*}$ \\
$\hat{A} L$ & $36.30(29.95-41.55)$ & $40.80(39.70-45.10)$ & $\mathbf{0 . 0 0 5 ^ { * }}$ \\
\hline
\end{tabular}

G1 (Female group), G2 (Male group), ÂC (Cervical angle), ÂT (Thoracic angle), ÂL (Lumbar angle).

Table 5 - Description of posture variables by age stratification.

\begin{tabular}{|c|c|c|c|}
\hline Variable & SB1 & SB2 & $p$ value \\
\hline$\hat{A} Q d$ & 25.79 (11.89) & $19.83(5.94)$ & $0.006 *$ \\
\hline ÂQe & $23.60(18.40-37.40)$ & $21.10(14.70-24.75)$ & $0.024 *$ \\
\hline$\hat{A} C$ & $40.60(34.80-44.00)$ & $34.10(31.80-35.45)$ & $0.001 *$ \\
\hline ÂT & $44.57(8.46)$ & $36.15(3.10)$ & $0.001 *$ \\
\hline
\end{tabular}

SB1 (Subgroup 10-12 years), SB2 (Subgroup 13-15 years), ÂQd (Right Q angle), ÂQe (Left Q angle), ÂC (Cervical angle), ÂT (Thoracic angle).

Table 6 - Description of postural balance variables.

\begin{tabular}{cccc}
\hline Variable & G1 & G2 & $p$ value \\
\hline CoP & $2.10(1.31-2.85)$ & $1.78(1.40-3.01)$ & 0.809 \\
OAP & $1.75(1.33-2.06)$ & $1.72(1.35-2.12)$ & 0.761 \\
OML & $1.54(1.15-1.96)$ & $1.60(1.27-2.06)$ & 0.562 \\
\hline
\end{tabular}

G1 (Female group), G2 (Male group), CoP (center of pressure of the body), OAP (oscillation in the anteroposterior direction), OML (oscillation in the mediolateral direction).

which occurs more frequently in male adolescents and young adult. ${ }^{(18)}$

Facing this scientific gap, this study was recommended, the specific examination that predicts the flexibility of the posterior trunk chain and directs the AS diagnosis. It was found that there was no difference between the groups, which presented a considerable decrease in mobility, predisposing the students to a probable AS medical picture in the future. Other study ${ }^{(19)}$ quantified the anterior flexibility index in adolescent growth spurt and also identified that there is no difference between girls and boys, even considering the particularities of each sex. In addition, there is a reduced and worrying level of mobility, taking into account the young age.

Faced with the technological age, the use of computers and smartphones have contributed to increasingly sedentary individuals, with significant muscle shortenings, which over time lead to postural deviations. ${ }^{(2-4)}$ Filipovic et al. ${ }^{(20)}$ tested the articular mobility of individuals with postural deviations, arguing that children with scoliosis would have a reduction in the physiological range of motion due to the muscular and postural compensations for maintaining the upright posture. 
This information allows us to speculate that the decrease in flexibility in this sample may be related to the high prevalence of scoliosis and the presence of scoliotic attitudes in the thoracic and lumbar level. In this variable, no difference was found between the male and female subjects, differing from the recent surveys that evidence the scoliosis being a postural alteration that affects more girls, ${ }^{(6,21,22)}$ which gives a different profile of the students studied in this study.

The weight of the school backpack is an aggravating factor that is often indicated as an important indicator of postural alterations and increase of the curvatures of the vertebral column, asymmetries in the craniovertebral, trunk and lower limbs segments and anterior displacement, causing children postural adaptations due to the adopted overload. ${ }^{(14,23)}$ However, in this study, the weight of backpacks in both groups did not present an overload characteristic, due to the reality of the public school, where there is a shortage of didactic materials, which could have a different proportion if our sample consisted of students from a private school.

The postural evaluation identified a significant difference between the groups, determining the overlap of valgus knee in females between 10 and 12 years of age, which coincidentally represents the phase of peak development of the girl. Our data corroborate with the recent meta-analysis of Holden et al. ${ }^{(24)}$, who investigated the existence of differences between the genders, regarding the postural variants of individuals in the phase of growth spurt and showed that due to skeletal maturation, female adolescents present valgus knee index greater in relation to males, although there is no consensus on sexual differences in postural stability.

Fulkerson et al. ${ }^{(25)}$ consider that the development of patellar changes in female is usually due to the greater hip width, allowing medial deviation of the femur and excessive lateral traction of the patella, estrogen action that allows patellar hypermobility, sitting crossing and adducting the legs causing internal rotation of the hip and use of shoes with high heels.

In lateral view, the position of the knee also reported a significant difference, with females showing hyperextension and males showng flexion pattern, in agreement with the findings of Dolphens et al ${ }^{(12)}$ who interpreted the knee semi-flexion posture in boys as a possible compensatory positioning strategy, used to correct the tendency of the anterior body imbalance. Although we consider the interpretations of the authors, we infer that this behavior is a consequence of the possible posterior chain shortening, mainly of the hamstring muscles, which in boys is predominant due to the strong relation with the practice of sports activities and the maintenance of the prolonged seated posture. ${ }^{(26,27)}$ And in the hyperextension in females, has been discussed the influence of loosening of the anterior cruciate ligament ( $A C L$ ) facilitated by menstrual cycle in the follicular period and luteal phase as a probable predictive condition. ${ }^{(28,29)}$

Our findings also point out significant differences between the sexes regarding the increase in vertebral curvatures. There was a marked development of the cervico-thoracic segment in boys (13 and 15 years) and lumbar hyperlordosis in girls (regardless of age). Factors such as postural inadequacy in the sitting position, computer use, physical activity, sleep hours exceeding 10 hours per night and transportation of school material were associated with the predisposition of kyphotic posture in adolescents of this age group. ${ }^{(8)}$ As for the increased lumbar curvature, Whitcome et al. ${ }^{(30)}$ reported that anatomic differences in relation to the size of the vertebra explain the high predisposition in females, which have a $14 \%$ greater vertebral body area and a $13 \%$ higher orientation of the articular surfaces in the coronal sense when compared to males. Thus, the female vertebral anatomy allows the spine to be better suited for greater load resistance and facilitates increased lordotic curvature.

Despite the differences found in the postural variations, no sexual differences were found in relation to the balance, which allowed us to find no correlation between posture and balance in this sample. We infer that postural changes developed in the growth phase are not capable of causing important body displacements, and this could be different in samples of adolescents with orthopedic or neurological dysfunctions and overweight or obesity profile, which suggests future research in this context.

\section{CONCLUSION}

The study suggests that the phase of corporal development in the school period favors postural phenotypic differentiations, in which the female adolescents present knee angles that exacerbate during 10 to 12 years and in the male the increase of the cervico-thoracic curvatures is evident between 13 and 15 years. Despite the high prevalence of scoliosis and reduced spine flexibility, no significant differences were found for backpack weight and balance, as well as correlation between posture and balance.

\section{LIMITATION OF STUDY}

The study determined broad thoracic and lumbar axial rotation by scoliometer did not getting data regarding the use of spinography imagen.

\section{AUTHOR'S CONTRIBUTION}

LKAN CBM FMNF- Collect the data, conceived and designed the experiments; FLC- Review the literature, wrote and corrected the article; MGRA- Designed the experimente and analysed the data; MBGJ - Guide the work, conceived and designed the experiments.

\section{CONFLICTS OF INTEREST}

The authors declare that there was no conflict of interest.

\section{AUTHOR DETAILS}

${ }^{3}$ Coordenator of Laboratory of Kinesiotherapy and Manual Therapy Resources, Federal University of Pernambuco, Universidade Federal de Pernambuco (UFPE), Recife (PE), Brazil.

${ }^{4}$ Coordenator of Motor Control and Postural Balance Laboratory, Federal University of Sergipe, Universidade Federal de Sergipe (UFS), Aracaju (SE), Brazil. 


\section{REFERENCES}

1. Rogol AD, Roemmich JN, Clark PA. Growth at puberty. Journal of Adolescent Health 2002;31:192-200.

2. Shehab DK, Jarallah KF. Nonspecific low-back pain in Kuwaiti children and adolescents: associated factors. Journal Adolescent Health 2005;36(1):325.

3. Straker LM, O’Sullivan PB, Smith A, Perry M. Computer Use and Habitual Spinal Posture in Australian Adolescents. Public Health Rep 2007;122(5):634-643.

4. Kee IK, Byun JS, Jung JK, Choi JK. The presence of altered craniocervical posture and mobility in smartphone-addicted teenagers with temporomandibular disorders. J Phys Ther Sci 2016;28(2):339-46.

5. Fonseca CD, Cardoso Dos Santos A, Candotti CT, Noll M, Luz AM, Corso CO. Postural education and behavior among students in a city in southern Brazil: student postural education and behavior. B J Phys Ther Sci 2015;27(9):2907-11.

6. Sakullertphasuk W, Suwanasri C, Saetang L, Siri N, Junsiri P, Yotsungnoen $\mathrm{S}$, et al. Prevalence of Scoliosis among High School Students. J Med Assoc Thai 2015;98(5):18-22.

7. Paananen MV, Auvinen JP, Taimela SP, Tammelin TH, Kantomaa MT, Ebeling HE. Phychosocial, mechanical, and metabolic factors in adolescents' musculoskeletal pain in multiple locations: a cross-sectional study. European Jounal of Pain 2010;4:395-401.

8. Sedrez JA, Rosa MIZ, Noll M, Medeiros FS, Candotti CT. Risk factors associated with structural postural changes in the spinal column of children and adolescents. Rev Paul Pediatr 2015;33(1):72-81.

9. Lafond D, Descarreaux M, Normand MC, Harrison DE. Postural development in school children: a cross-sectional study. Chiropr Osteopat 2007;15:1.

10. Mac-Thiong J-M, Labelle H, Roussouly P. Pediatric sagittal alignment. Eur Spine J 2011;20(5):586-590.

11. Dolphens $M$, Cagnie B, Vleeming A, Vanderstraeten G, Coorevits $P$, Danneels L. A clinical postural model of sagittal alignment in young adolescents before age at peak height velocity. European Spine Journal. 2012;21(11):2188-2197.

12. Dolphens M, Cagnie B, Vleeming A, Vanderstraeten G, Danneels L. Gender differences in sagittal standing alignment before pubertal peak growth: the importance of subclassification and implications for spinopelvic loading. J Anat 2013;223(6):629-640.

13. Neuschwander TB, Cutrone J, Macias BR, Cutrone S, Murthy G, Chambers $\mathrm{H}$, et al. The effect of backpacks on the lumbar spine in children: a standing magnetic resonance imaging study. Spine 2010;35(1):83-8.

14. Walicka-Cupryś K, Skalska-Izdebska R, Rachwał M, Truszczyńska A Influence of the Weight of a School Backpack on Spinal Curvature in the
Sagittal Plane of Seven-Year-Old Children. BioMed Research International 2015;6.

15. Drza-Grabiec J, Snela S, Rykała J, Podgórska J, Rachwal M. Effects of the sitting position on the body posture of children aged 11 to 13 years. Work 2015;51(4):855-62.

16. World Health Organization (WHO). Adolescent health and development 2007. Disponível em: http://www.who.int/child-adolescent-health/.

17. Ferreira EAG, Duarte M, Maldonado EP, Bersanetti AA, Marques AP. Quantitative Assessment of Postural Alignment in Young Adults Based on Photographs of Anterior, Posterior, and Lateral Views. Journal of Manipulative and Physiological Therapeutics 2011;34(6):371-380.

18. Tsirikos AI, Garrido EG. Spondylolysis and spondylolisthesis in children and adolescents.J Bone Joint Surg Br 2010;92:751-759.

19. Lamari NM, Marino LHC, Cordeiro JA, Pellegrini AM. Trunk anterior flexibility in adolescents after height growth speed peak. Acta ortop. bras 2007;15(1).

20. Filipovic V, Stalec NV. The Mobility Capabilities of Persons With Adolescent Idiopathic Scoliosis. Spine 2006;31(19):2237-2242.

21. Zhang H, Guo C, Tang M, Liu S, Li J, Guo Q, et al. Prevalence of scoliosis among primary and middle school students in Mainland China: a systematic review and meta-analysis. Spine 2015;40(1):41-9.

22. Baroni MP, Sanchis GJ, De Assis SJ, dos Santos RG, Pereira AS, Sousa KG, et al. Factors associated with scoliosis in schoolchildren: a crosssectional population-based study. J Epidemiol 2015;25(3):212-20.

23. Ramprasad M, Alias J, Raghuveer AK. Effect of backpack weight on postural angles in preadolescent children. Indian Pediatr 2010;47(7):575-80.

24. Holden S, Boreham C, Delahunt E. Sex Differences in Landing Biomechanics and Postural Stability During Adolescence: A Systematic Review with Meta-Analyses. Sports Med 2016;46(2):241-53.

25. Fulkerson JP, Arendt EA. Anterior knee pain in females. Clin Orthop 2000;372:69- 73.

26. Coelho JJ, Graciosa MD, Medeiros DL, Pacheco SCS, Costa LMR, Ries LGK. Influence of flexibility and gender on the posture of school children. Rev Paul Pediatr 2014;32(3):223-228.

27. Veiga PH, Daher CR, Morais MF. Postural alterations and flexibility of the posterior chain in soccer's injuries. Rev Bras Cienc Esporte 2011;33:235-48.

28. Shultz SJ, Sander TC, Kirk SE, Perrin DH. Sex differences in knee joint laxity change across the female menstrual cycle. J Sports Med Phys Fitness 2005;45(4):594-603.

29. Dedrick GS, Sizer PS, Merkle JN, Hounshell TR, Robert-McComb JJ, Sawyer $\mathrm{SF}$, et al. Effect of sex hormones on neuromuscular control patterns during landing. J Electromyogr Kinesiol 2008;18(1):68-78.

30. Whitcome KK, Shapiro LJ, Lieberman DE. Fetal load and the evolution of lumbar lordosis in bipedal hominins. Nature 2007;450:1075-1078. 